\title{
Rhinovirus A
}

National Cancer Institute

\section{Source}

National Cancer Institute. Rhinovirus A. NCI Thesaurus. Code C112407.

A species of non-enveloped, spherical viruses with a capsid with quasi $T=3$ icosahedral symmetry in the Picornaviridae family and Enterovirus genus. The genome is composed of single-stranded, positive-sense RNA. Rhinovirus A is found in humans and causes respiratory infections and the common cold. Rhinovirus $A$ is transmitted via direct person-to-person contact and aerosols of respiratory droplets. 